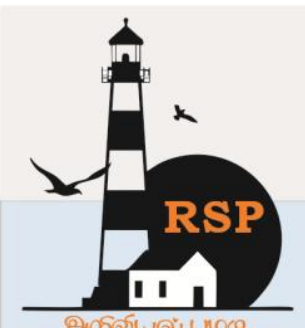

INTERNATIONAL RESEARCH JOURNAL ON ADVANCED SCIENCE HUB

ISSN : $2582-4376$
Open Access

RSP SCIENCE HUB

(The Hub of Research Ideas)

Available online at www.rspsciencehub.com

Special Issue of Second International Conference on Advancements in Research and Development (ICARD 2021)

\title{
OCR for Medical Data Processing
}

Mathumitha $I^{1}$, Vinodhini $B^{2}$, Jayaprakash $S^{3}$, Malini Devi $R^{4}$, Sangeetha $K^{5}$

${ }^{1,2,3}$ UG Scholar, Department of Computer Science and Engineering, SNS College of Technology, Coimbatore, India.

${ }^{4,5}$ Assistant Professor, Department of Computer Science and Engineering, SNS College of Technology, Coimbatore, India.

mathuim999@gmail.com ${ }^{1}$,profbvinodhini1975@gmail.com ${ }^{2}$, sjayaprakash@gmail.com ${ }^{3}$

malinidevippy@gmail.com ${ }^{4}$,Sangithaprakash@gmail.com ${ }^{5}$

\begin{abstract}
The mechanism which implement in this device is to help visual impairment people and also to modify the hardcopy hospital data into softcopy. The modified softcopy file will be stored as a backup in the drop box. In this project, a device which developed by a web is converts an image text to speech. The basic structure of the system is to capture an image, extract only the interest region (i.e. The image region which has text) and converts that text speech. The device is implemented with the help of system and a web camera. It locates the parts of images which contains the text and eliminate the background by various image pre-processing from the captured image. Two tools are required to convert the captured image (which contains text only).
\end{abstract}

Keywords: Optical Character Recognition, Text To Speech, Image to Text and Audio.

\section{Introduction}

In our world of 7.8 billion humans, 2.2 billion people are visually impaired out of which, one billion people cases could have barred or have been absent unaddressed(WHO,2019).In on a daily basis routine, reading is a major importance for humankind. For the duration of understanding a lot of complications were faced by visually impaired people. Our machine which assist the people who are visually impaired by analysis out the text towards them. There is a variety of kind helping method for visually impaired to read. The live technologies use a relatively move towards as described in this paper, but they have exact drawbacks. Firstly, the key images which have been taken from earlier works have no obscure backdrop i.e., the text inputs are in print on a plain white sheet. It is convenient to transfer such image to text not including pre-processing, but such a advance will not be favourable in a factual time. We required machine which able to locate out out the text from any obscure background and read it powerfully. Being able to notice an area enclosing by four points, we imagine that this is a necessary area containing the text. It undergoes edge detection after new image obtained and a precinct is than draw over the letter. The OCR and TTS processed the image to give audio output.

\subsection{Need for TTS and OCR}

The conversation of images of typed, handwritten or printed text into machine coded text by OCR which is mechanical or electronically conversation. The OCR Technology is to automatically scan the texts which present in the document convert it into a text searchable document. A text to speech (TTS) synthesizer is a computer-based device that read text aloud mechanically, not considering of whether the test is introduced the device that can 
read text audibly mechanically, apart from of whether the is introduced by a PC input stream or a scanned key submitted to implemented by equally hardware and software. Speech is frequently based concatenation of usual speech i.e. Units that are occupied from ordinary speech all mutually to outline a word or sentence.[1-5]

\subsection{Machine Learning}

Machine Learning is the region to give knowledge in computers the potential to learn without being clearly planned. Machine Learning is one of the electrifying technologies that one would have ever come diagonally. Machine Learning algorithms are widely used in various kinds of applications such as email filtering and computer vision, where it is difficult or unfeasible to develop conventionally algorithms to perform the needed tasks. Machine Learning engineers started to explore existing OCR APIs and SDKs.To reduces some of OCR shortcomings machine learning OCR uses AI technologies. To help preprocess by the usage of OCR. It can handle the document complexity remains limited and still templates are used.[6-9]

\section{Literature Survey}

Sangam Borkar, Nidhi Kalidas Sawant "written Text to Speech Conversion using OCR". Text to speech is very a lot helpful in today's globe. People make use of this knowledge in their everyday occupation but couldn't understand it. A text-tospeech (TTS) scheme transforms ordinary words text into speech sign. In this document Devanagari text to speech alteration is ended for Marathi on paper text. To reach the mandatory output the two techniques are implemented that are Optical Character Recognition (OCR) and Text to Speech (TTS) system. OCR is utilized to alter the text from an image into editable text which is done using multiclass maintain Vector Machine (SVM) and Text to Speech mechanism gives the audio output. J.J.Mullani,M.Sankar,Priyanka.SKhade,Snehal.HS onalkar,Nikita.LPatil"OCR for speech synthesized system", Optical character acceptance has twisted out to be a stick out in the midst of the best utilizations of modernization in the ground of example acceptance and artificial intelligence. In present culture, there is an amazing call for to quickly consist of generous quantify of printed and by hand written data into the PC, by the side of these lines every person depend dynamically on PCs to process incredible volumes of in order. The vital aim is to allow vocally out of action persons to make use of the PC or to check documentation in a simpler technique the structure is detached into two sections initially is Optical Character Recognition (OCR) and second part is content to discussion. In the initial segment, Virtual device is shaped in which a hued image that contains the font is transformed above into gray scale image and font are arranged and in the second part; conversion from content to discussion is shaped. The signify of standard review time, model variation, slightest test time and most tremendous evaluation time in $\mathrm{ms}$ is approximate. There are a not many varieties in moment parameters due to factors similar to digit of font seeming, line outline, histogram, glow, dissimilarity and gamma reconsideration esteems.

K.Karthick, K.B.Ravindrakumar, R.Francis, S.Ilankannan "Text Recognition and Recent Research in OCR". The Optical Character Recognition (OCR) is one of the mechanical recognition techniques that complete the mechanization wants in different applications. A engine can examine the information current in ordinary scenes or other equipment in any shape with OCR. The typed and in print character recognition is basic due to its definite size and figure. The script of persons differs in the on top of aspect. So, the hand written OCR method faces complication to learn this variation to identify a character. In this document, we discussed the various stages in text detection, handwritten OCR systems categorization according to the text kind, study on Chinese and Arabic text detection as well as use oriented current study in OCR.[5-11].

\section{Proposed System}

To conquer the trouble in the active system to have developed a mission for sightless People by means of neural OCR in Open CV. The proposed system is to help blind persons to read text from demanding patterns and backgrounds for the reason of reading documents. The major aim of device is to recognize the text in the papers. Firstly, the article image is captured by using a webcam which is fixed within System and is followed by the image giving out. To accomplish a mechanized system, which checks a article and reads out its material to the personage on click of a key? The vocal is delivered with the support of a speaker which would aid the personage to read aloud the content in the scan paper. System helps the blind people for the reason of reading not 
including consuming much gap. The Documents that are red will be uploaded in the drop box for added upcoming procedure.

\section{Block Diagram}

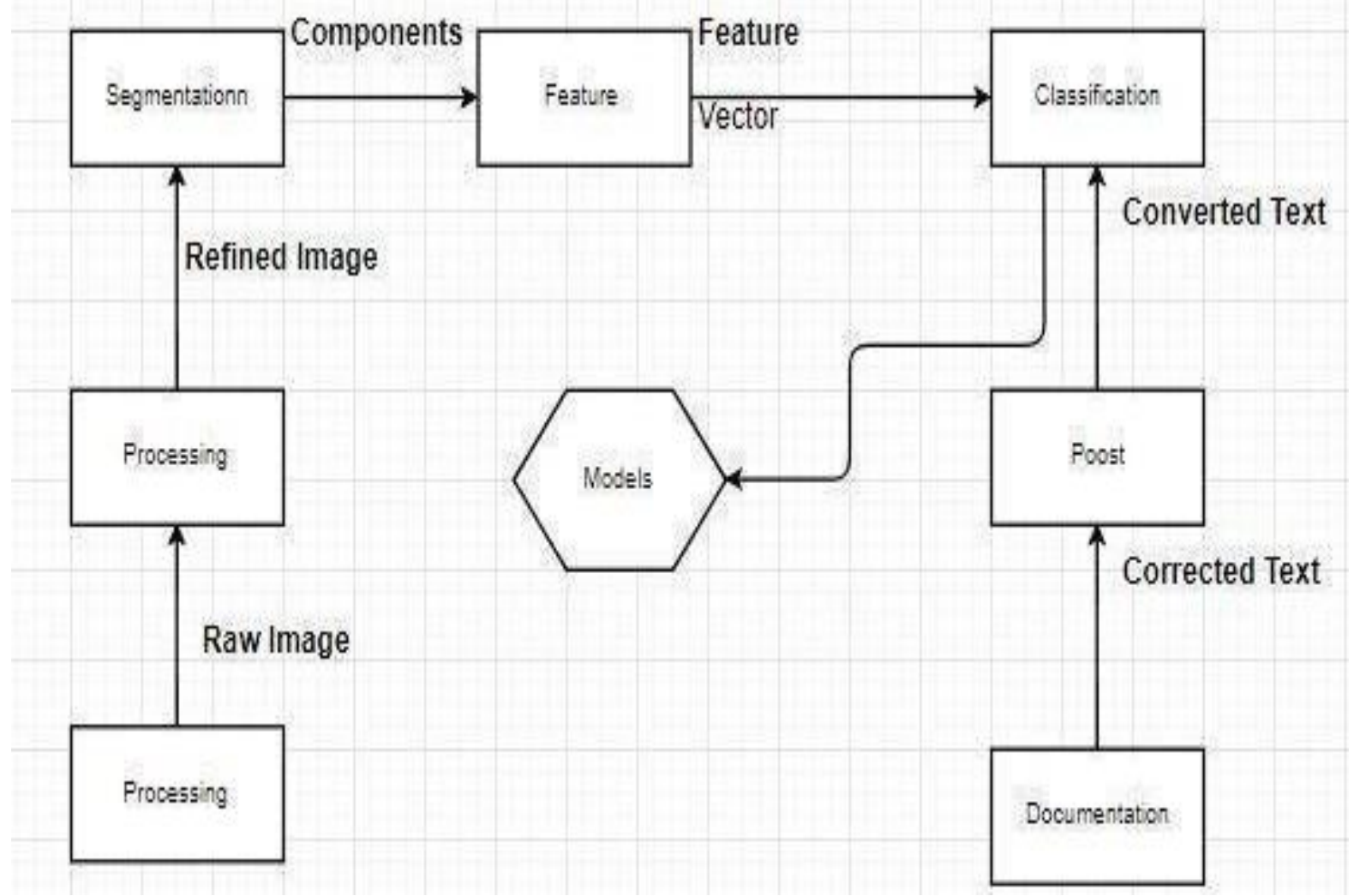

Fig 1.Image Classification

\section{Conclusion}

This machine is a performance of text to speech exchange for sightless and visually impaired people. Our algorithm processed beneficially and reads the text powerfully. Establishment the hardware performance with mixture of samples. The gadget is compacted and able to locate the books in records and permit the repossession of the read documents. Thus, we have designed a multifunctional system, which acts as an support for striking the excellence of one's living. This project is through upon by different platforms such as python with different packages which is automatic and run on device by establishing this device. We are converting image to text to speech, personnel assistant based on Google with home mechanization. Amazon Echo was unsuccessful to suggest the user with a sense of control as it occasionally remained insensible when Google voice instructions that are not valid when practical as personnel assistants and these sort of personnel assistance are fashionable.

\section{Future work}

The system will lead the future by recorded system which needed to help for visually impaired people to listen and also the personnel health record is scanned as a document by using OCR, stored in the drop box, it will handle for the future people to know their health record instead of handwritten medical records databases. Machine Learning takes a major place in the device, so it can reach a great development to help the visually impairment people at the same time storage of personnel record for visually impaired people. Also, this technology machine learning with OCR will help more in the areas of medical field and also for storage of records for many fields in necessary storage area.

\section{Application}

1. Office Lens is the most advanced application developed by OCR to convert to image to text.

2. Smart Lens application help to convert image to text and also helps to convert translate text into various languages. 
3.Adobe OCR is one of the most popular applications when it comes for managing documents by scanning and detecting automatically.

\section{References}

[1].M.cai, J. Song and M.R.Lyu.A New Approach for video Text Detection. In proc. of International Conference on Image Processing, Rochester, New York, USA, pp.117-120, 2002.

[2].C.Patel, A.Patel, D.Patel,'Optical Character Recognition by Open Source OCR Tool Tesseract: A Case Study", International Journal of Computer Applications, vol. 55, no. 10.October 2012.

[3].https://www.hacksteer.io/rgrokett/Webtextread er-for-impaired-vision-695331.

[4].https://ieeexplore.ieee.org/abstract/document/7 919526/

[5].http://ijarcsse.com/Before_August_2017/docs/ papers/Volume_7/7_July2017/V717-0117.pdf.

[6].S.Musale, V.Ghiye,"smart reader for visually impired", $20182^{\text {nd }}$ International Conference on Inventive Systems and Control (ICISC), 2018.

[7].A.Manikandan, S.Choudhury, S.Majumder,"Text reader for visually impaired people: Any reader", 2017 IEEE International Conference on Power Control Signal and Instrumentation Engineering (ICPCSI), 2017.

[8].S.Sabab, M.Ashmafee,"Blind Reader: An intelligent assistant for blind", $201619^{\text {th }}$ International Conference on Computer and Information Technology (ICCIT), 2016.

[9].Pardeep Kaur, Pooja Choudhary, "Review on: English Scanned Documents", International Journal of Engineering Research-Online, vol. 3, no. 2, pp. 60-65, 2015.

[10].Sarkar Prateek, Henry S. Baird, Xiaohu Zhang, "Training on severely degraded textline images", Proceedings of the International Conference on Document Analysis and Recognition, vol. I, pp. 38-43, August 2003.

[11].Kristen Summers, T. Kanungo, E. H. B. Smith, J. Hu, P. B. Kantor, "Document image improvement for OCR as a classification problem", Proceedings of SPIE/IS\&T Electronic Imaging Conference on Document Recognition \& Retrieval $X$, vol. 5010, pp. 7383, January 2003. 